\title{
Second-harmonic scanning optical microscopy of poled silica waveguides
}

\author{
Pedersen, Kjeld; Bozhevolnyi, Sergey I.; Arentoft, Jesper; Kristensen, Martin; Laurent-Lund, Christian
}

Published in:

Journal of Applied Physics

Link to article, DOI:

$10.1063 / 1.1290261$

Publication date:

2000

Document Version

Publisher's PDF, also known as Version of record

Link back to DTU Orbit

Citation (APA):

Pedersen, K., Bozhevolnyi, S. I., Arentoft, J., Kristensen, M., \& Laurent-Lund, C. (2000). Second-harmonic scanning optical microscopy of poled silica waveguides. Journal of Applied Physics, 88(7), 3872.

https://doi.org/10.1063/1.1290261

\section{General rights}

Copyright and moral rights for the publications made accessible in the public portal are retained by the authors and/or other copyright owners and it is a condition of accessing publications that users recognise and abide by the legal requirements associated with these rights.

- Users may download and print one copy of any publication from the public portal for the purpose of private study or research.

- You may not further distribute the material or use it for any profit-making activity or commercial gain

- You may freely distribute the URL identifying the publication in the public portal

If you believe that this document breaches copyright please contact us providing details, and we will remove access to the work immediately and investigate your claim 


\title{
Second-harmonic scanning optical microscopy of poled silica waveguides
}

\author{
Kjeld Pedersen a) and Sergey I. Bozhevolnyi \\ Institute of Physics, Aalborg University, Pontoppidanstrcede 103, DK-9220 Aalborg, Denmark \\ Jesper Arentoft, Martin Kristensen, and Christian Laurent-Lund ${ }^{\text {b) }}$ \\ Research Center COM, Technical University of Denmark, Building 349, DK-2800 Lyngby, Denmark
}

(Received 6 March 2000; accepted for publication 6 July 2000)

\begin{abstract}
Second-harmonic scanning optical microscopy (SHSOM) is performed on electric-field poled silica-based waveguides. Two operation modes of SHSOM are considered. Oblique transmission reflection and normal reflection modes are used to image the spatial distribution of nonlinear susceptibilities in the sample surface plane and in depth. It is shown that the spatial resolution in normal reflection mode can be better than $1 \mu \mathrm{m}$ for second-harmonic images. A simple qualitative description of this operation mode is suggested and found to be in good agreement with the results obtained. Advantages and limitations of the two operation modes when used for SHSOM studies of poled silica-based waveguides are discussed. The influence of surface defects on the resulting second-harmonic images is also considered. (C) 2000 American Institute of Physics.
\end{abstract}

[S0021-8979(00)10619-X]

\section{INTRODUCTION}

Second-harmonic generation (SHG), being extremely sensitive to the symmetry of materials, ${ }^{1}$ is widely used as a nondestructive and noncontact probe of surfaces and interfaces. ${ }^{2}$ Furthermore, starting with the first experiments on spatially resolved SHG from a dye monolayer, ${ }^{3}$ SHG microscopy has evolved into a very useful characterization tool for inhomogeneous surfaces. It has recently been applied to imaging of periodically poled ferroelectric domains ${ }^{4}$ and domain walls, ${ }^{5}$ domain structures in epitaxially grown magnetic garnet films ${ }^{6}$ and Langmuir-Blodgett monolayers, ${ }^{7}$ and polar orientational distribution in thin polymer films. ${ }^{8}$ Poled silicabased waveguides are yet another interesting objective for SHG microscopy that allows one to map spatial variations of second-order optical nonlinearities (SONs) and, thereby, to study the mechanisms of poling as well as to characterize the poled components. ${ }^{9}$ Note that, contrary to projection type SHG microscopy, ${ }^{4-7}$ the scanning technique ${ }^{8,9}$ allows investigations of large areas and, subsequently, precise selection of smaller regions for detailed inspection, always keeping the same intensity scale over the whole image.

In this article, we present the results of detailed investigations of second-harmonic scanning optical microscopy (SHSOM) of poled silica-based waveguides. We consider two different operation modes of SHSOM and demonstrate their usage for imaging of SON variations both along the sample surface plane and in depth. Advantages and limitations of these modes when used for SHSOM studies of poled silica-based waveguides are discussed. Finally, we report on SH images of surface defects and discuss their influence on the resulting SH images of SON in poled waveguide structures.

\footnotetext{
a) Corresponding author; electronic mail: i13kp@physics.auc.dk

b)Present address: IONAS A/S, CAT Bldg. 347, Technical University of Denmark, DK-2800 Lyngby, Denmark.
}

\section{EXPERIMENT}

The setup used for the experiments is illustrated in Fig. 1. It is constructed as a scanning optical microscope that allows detection of both the fundamental-harmonic (FH) and the generated SH frequency in the reflected light. The pump light is delivered by a mode-locked Ti:sapphire laser tunable in the range of $720-900 \mathrm{~nm}$ with a pulse length of $\sim 200 \mathrm{fs}$. The light is focused onto the sample by a microscope objective or a single lens. The sample is scanned under the focused beam by stepper motor driven stages (calibrated with measurements on a diffraction grating). At normal incidence, the reflected light is collimated as it is returned through the focusing lens, and it is then reflected into the detectors by a $50 \%$ beamsplitter [Fig. 1(a)]. A small fraction of the light is reflected from a glass plate into a photodiode in order to record the FH radiation, i.e., the linear reflectivity of the sample. The remaining part is passed through a filter to absorb the pump light before detection of the SH radiation by a photomultiplier tube. Generation of the SH field polarized perpendicular to the sample surface can be probed by rearranging the setup to oblique incidence of the incident light beam (e.g., $40^{\circ}$ ). An additional lens is then introduced in order to collimate the reflected $\mathrm{FH}$ and $\mathrm{SH}$ radiation [Fig. $1(\mathrm{~b})]$.

The samples consisted of three layers of glass deposited by plasma enhanced chemical vapoer deposition on $n$-type doped silicon wafers [Fig. 1(b)]. The bottom and top (cladding) layers were pure $\mathrm{SiO}_{2}$, while the central (core) layer consisted of Ge-doped $\mathrm{SiO}_{2}$ codoped with either $\mathrm{Al}$ (sample S1) or N (sample S2 and S3). ${ }^{10}$ The thickness of the layers given in the caption of Fig. 1 was estimated from scanning electron microscope data. The wafers were cleaved into samples $\sim 2.5 \mathrm{~cm} \times \sim 2.5 \mathrm{~cm}$. Conductive glue was painted onto the top of sample $\mathrm{S} 1$ while $\mathrm{Al}$ was evaporated onto top of the glass of samples S2 and S3. 

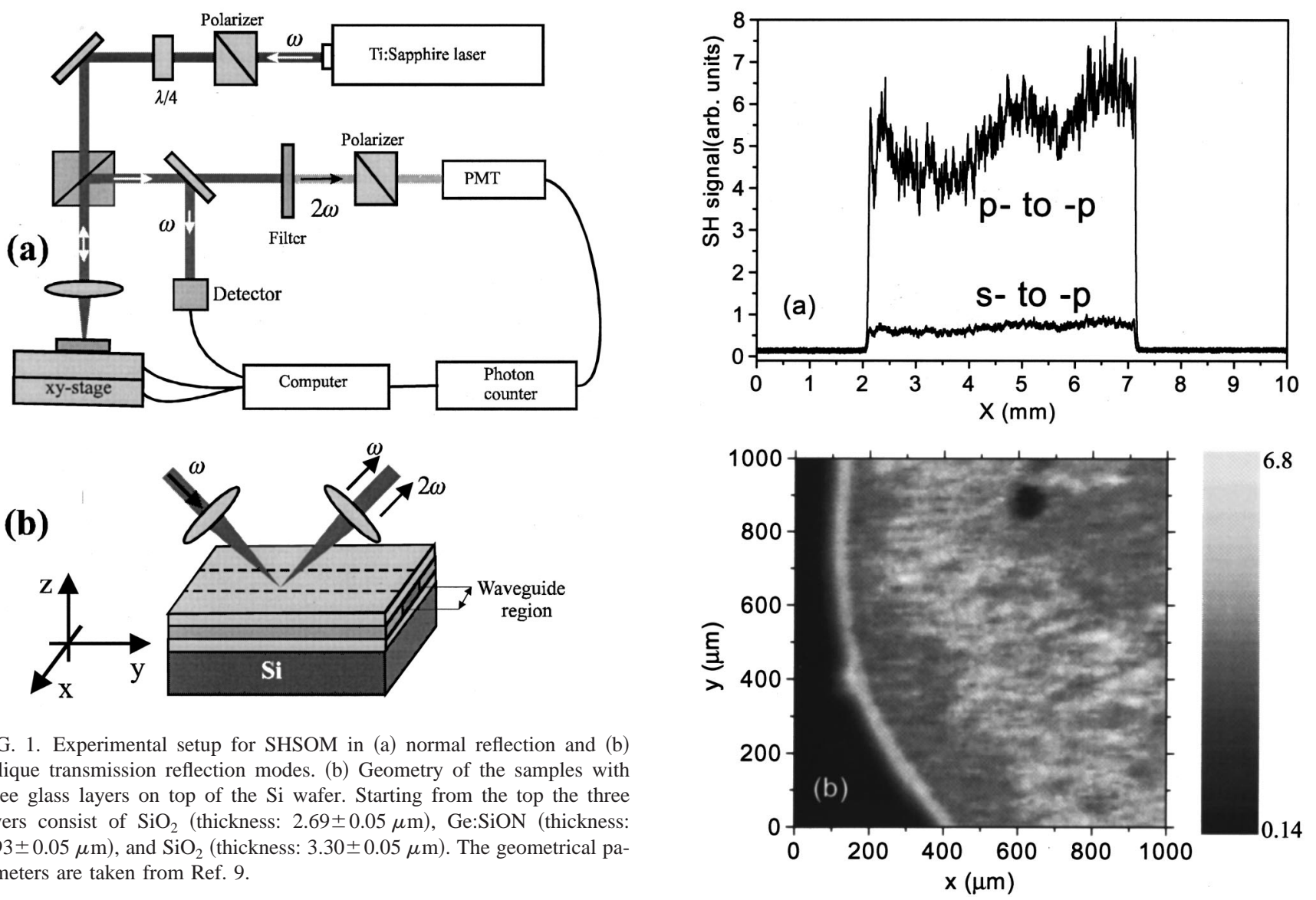

Sample S1 was placed on a heater and brought to $280{ }^{\circ} \mathrm{C}$. The sample was poled by applying $300 \mathrm{~V}$ to a round pad formed by the homogeneous (i.e., without grooves) conductive glue layer and keeping the silicon wafer grounded. Before removing the high voltage, the sample was cooled to room temperature. Sample S2 (used in our previous experiments $^{9}$ ) was poled by applying $1.5 \mathrm{kV}$ to the Al layer, which represented a photolithographically fabricated mask with grooves of $10 \mu \mathrm{m}$ width, and simultaneous exposure to $\mathrm{CO}_{2}$ laser radiation $(10.6 \mu \mathrm{m})$. The silicon wafer was grounded. With this procedure the glass regions below the grooves in the $\mathrm{Al}$ mask were exposed to radiation and to a somewhat inhomogeneous electric field. The same mask was then used for the fabrication of ultraviolet (UV)-induced channel waveguides (needed to measure the linear electrooptic effect). ${ }^{9}$ Sample S3 (also used in our previous experiments $^{9}$ ) was poled using the same procedure as that described for S1. However, the poling temperature was $400{ }^{\circ} \mathrm{C}$, and $2 \mathrm{kV}$ was applied to a rectangular (photolithographically defined) pad of the homogeneous Al layer.

\section{OBLIQUE TRANSMISSION REFLECTION MODE}

Silica-based components are produced through complicated processes involving, e.g., thin film deposition, annealing, UV writing, and poling. ${ }^{9,10}$ In order to optimize their performance, it is important to carry out spatially resolved characterization of the components. SH imaging conducted from the top surface provides an overview of the resulting spatial distribution of SON induced during the poling pro-

FIG. 2. (a) Cross section of the SH images (for two polarization configurations) through the poled region of sample $\mathrm{S} 1$ and (b) $\mathrm{SH}$ image (for $p$-to- $p$ polarization configuration) of a $1 \times 1 \mathrm{~mm}^{2}$ area of the boundary between the poled and unpoled regions. The signals were obtained at a $40^{\circ}$ angle of incidence with an $8 \times$ objective.

cess. Since the poling field creates a preferred direction along the surface normal, the SH field component polarized normal to the surface is expected to be the strongest one. Actually, in the approximation of homogeneous poling field (and sample) and for plane-wave illumination, SHG should only be observed under oblique incidence as $p$-polarized light (the electric field in the plane of incidence) and not as $s$-polarized light (the electric field perpendicular to the plane of incidence). With the scanning technique, large areas (e.g., 1 $\times 1 \mathrm{~cm}^{2}$ ) can be tested before smaller areas are selected for more detailed investigations. Figure 2 shows a onedimensional scan through the poled area of sample S1 and a section of the boundary between the poled and unpoled regions recorded at a $40^{\circ}$ angle of incidence [Fig. 1(b)]. The electrode, which was removed before the SH imaging, determines the poled region, in this case a circularly shaped area. The fluctuations in SHG seen in Fig. 2(a) are much larger than the experimental noise. Furthermore, the strong correlation between the fluctuations observed in the scans recorded in the same area with $p$ - and $s$-polarized pump light, respectively, demonstrates that the signal variations reflect the properties of the sample. The slow variation in the $\sim 1 \mathrm{~mm}$ range could be caused by inhomogeneities in the poling field and/or variations in the chemical composition of the glass. 

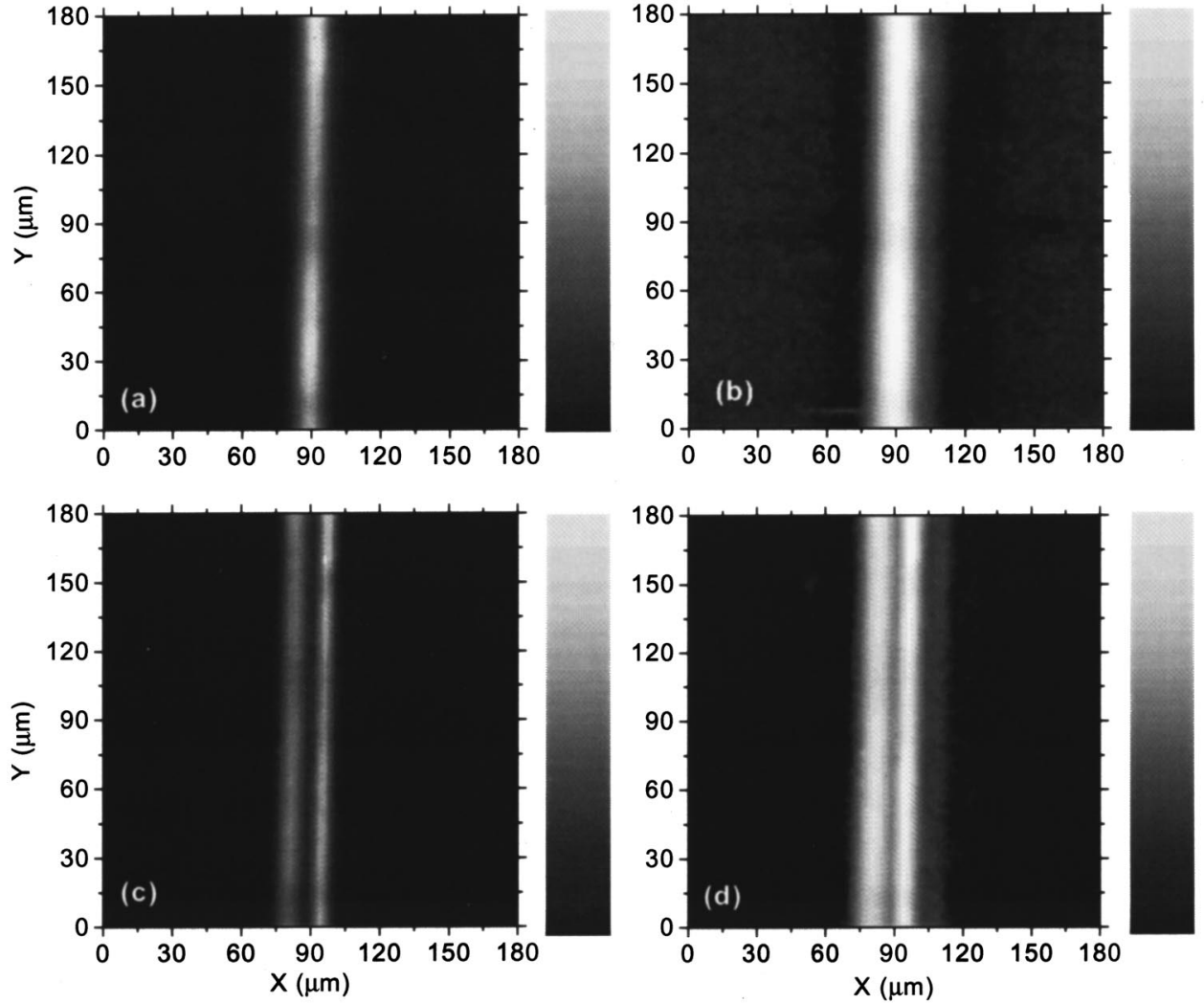

FIG. 3. SH images of an UV-written waveguide in sample $\mathrm{S} 2$ recorded with (a), (b) $p$-to- $p$ and (c), (d) $s$-to-s polarization configurations. The images in (a) and (c) are displayed in a logarithmic scale in (b) and (d), respectively, in order to enhance details at low intensity. The contrast in linear images (a), (c) is $100 \%$ and the ratio between their maximum signals is $\sim 5$. The images were obtained at a $40^{\circ}$ angle of incidence with an $8 \times$ focusing objective.
Figure 2(b) shows an example of a defect in the electrode leading to a large unpoled region $[$ at $(x, y)=(600,900)]$. Other effects such as local scattering of light can give rise to faster spatial variations in SHG. The image in Fig. 2(b) shows that special effects appear at the border of the poled area where a strong poling is observed in a $30 \mu \mathrm{m}$ wide region. These effects are probably caused by the complicated distribution of the electrostatic poling field at the edge of the electrode.

Applications of poled glasses for integrated optical components usually require that channel waveguides are defined in the glass layer. Figure 3 shows the SH images of a 180 $\times 180 \mu \mathrm{m}^{2}$ section of a UV-written waveguide in sample S2. The images were recorded for the $p$-to- $p$ and $s$-to- $s$ polarization configurations and with the channel waveguide oriented parallel to the plane of incidence (see Fig. 1). Here $p$ to $p$ and $s$ to $s$ refer to fundamental-to-SH polarizations. The width at half maximum of the $p$-to- $p$ signal peak is $12 \mu \mathrm{m}$, which is consistent with the $10 \mu \mathrm{m}$ wide openings in the $\mathrm{Al}$ electrode. ${ }^{9}$ In the $\log$ scale it is clearly seen that the $p$-to- $p$ signal has minima along the edges of the poled region [Fig. 3(b)]. This feature can be attributed to destructive interference between the background SH signal (e.g., from interfaces) and the signal from the poled glass region. Note that the reduced SH signal at the edges makes the center peak appear too narrow. ${ }^{11}$ A more detailed study of the width of the poled region would require a convolution fit of the SON distribution and the beam profile. Such an analysis is demonstrated for the depth profiles in Sec. IV, but will not be performed here since there is no independent measurement of the beam profile. The apparent asymmetry seen in the images represented in the log scale [Figs. 3(b) and 3(d)] is most probably related to the fact that it is very difficult to adjust the plane of incidence of the interrogating beam so that it will be exactly parallel to the channel waveguide.

The images in Figs. 3(c) and 3(d) recorded with $s$-polarized $\mathrm{SH}$ radiation show a structure with a minimum at the center of the waveguide. Note that the $s$-to- $s$ signal indicates that the symmetry in the surface plane is broken. ${ }^{2} \mathrm{We}$ believe that inhomogeneities in the poling field at the openings in the $\mathrm{Al}$ electrode are responsible for the detected signal and the SH images obtained. Indeed, the $x$ component of the poling field, which can result in in-plane poling of the glass (leading to $s$-polarized SH light), should be strongest at the edges of the openings and vanish at the center.

In order to get quantitative information from the $\mathrm{SH}$ scans, the angle of incidence has to be considered since this determines the relative importance of different SON tensor elements. Figure 4 shows the $\mathrm{SH}$ signal from sample S3 as a function of angle of incidence for the $p$-to- $p$ and $s$-to- $p$ polarization configurations along with an illustration of the geometry of SHG in the transmission reflection mode. Effects of local variations in the $\mathrm{SON}$ on the $\mathrm{SH}$ signal have been reduced by focusing the pump laser beam with a long focal length $(300 \mathrm{~mm})$ lens. A rather irregular variation of the $\mathrm{SH}$ signal with the angle, especially for the $s$-to- $p$ polarization configuration, is observed. This can be qualitatively accounted for as follows. Let us consider SHG in this geometry as the sum of contributions from dipole sheets making up the SON (poled) layer, as described by Sipe. ${ }^{12}$ Each dipole sheet generates $\mathrm{SH}$ radiation in the forward (transmission) and backward (reflection) directions. However, the accumulated 


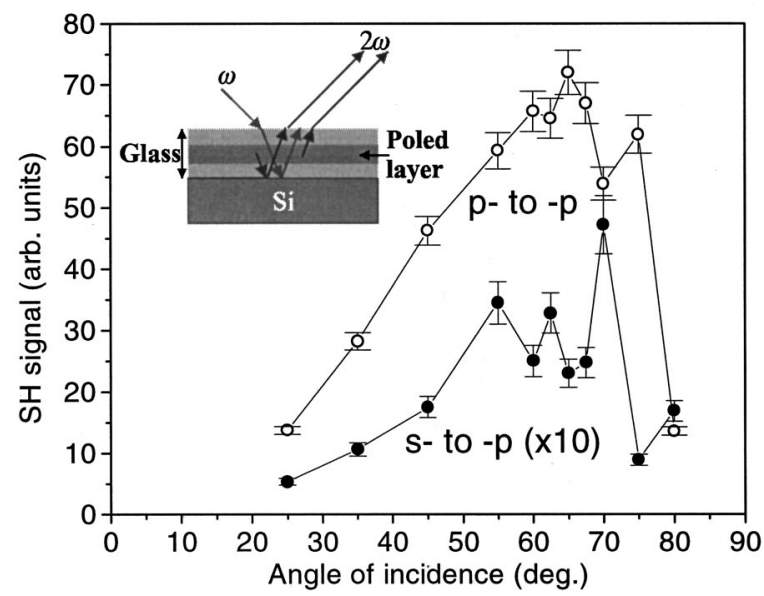

FIG. 4. Dependence on angle of incidence of the SH signals obtained in the $p$-to- $p$ and $s$-to- $p$ polarization configurations with sample S3. The inset illustrates SHG in the transmission reflection mode.

SH radiation in the backwards direction from the poled layer turns out to be negligible compared to that in the forward direction for the thickness (larger than $1 \mu \mathrm{m}$ ) of the considered SON layer. ${ }^{9}$ The reason is that, for thick layers, the phase matching condition for SHG in the direction perpendicular to the layer becomes progressively important, resulting in attenuation of the (nonsynchronized) SH radiation in reflection. ${ }^{13}$ Thus, the designation transmission reflection mode has been chosen for this configuration since the $\mathrm{SH}$ radiation is generated in transmission (through the poled layer) from the incident $\mathrm{FH}$ radiation and the $\mathrm{FH}$ radiation reflected at the glass/Si interface (see the inset in Fig. 4). Furthermore, multiple reflections of both the fundamental and the SH light at the boundaries of the glass layer have to be taken into account. It turns out that multiple reflections of the $\mathrm{FH}$ radiation are the most important. The oscillations in the SH signal (Fig. 4) are therefore related to the configuration of the standing wave interference pattern for FH radiation or, more precisely, to the (angular dependent) position of the FH intensity (local) maximum with respect to the SON layer.

The same reasoning can be used to explain why the oscillations in Fig. 4 are more pronounced for the $s$-polarized pump light. The SH signals have their maxima in the range of incidence angles being between $55^{\circ}$ and $75^{\circ}$. In this range, the internal glass/air reflection coefficient for $p$ polarization is much lower than for $s$ polarization because of the proximity to the Brewster angle. The contrast of the standing wave interference pattern is thus notably larger for the $s$-polarized FH radiation than for the $p$-polarized one. Clearly, the extraction of quantitative information about the poled material requires knowledge of the geometrical parameters of the structure. Furthermore, detailed angular dependent measurements of the SH signal at the same place on a sample (or SH imaging at different angles of incidence) are necessary. However, it is difficult to vary the incidence angle without shifting the pump beam on the sample and thus changing the probed area. This leads to problems with interpretation of data from inhomogeneous systems. Overall, we find the oblique transmission reflection mode rather convenient for
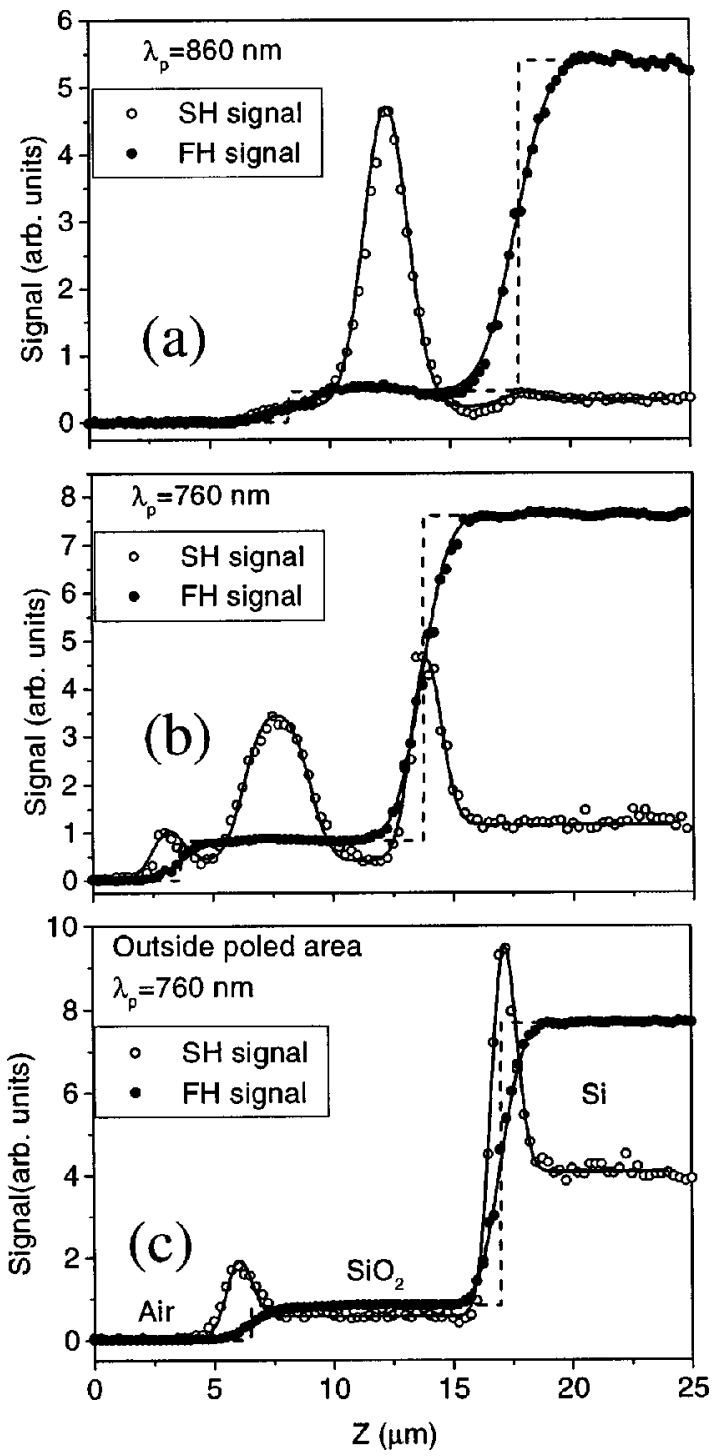

FIG. 5. Depth SH scans on a cleaved face of sample S3. The scan in (a) was recorded with $860 \mathrm{~nm}$ pump light, while those in (b) and (c) were recorded at $760 \mathrm{~nm}$. The scans in (a) and (b) were taken at different places in the poled region while the scan in (c) was taken outside the poled region.

qualitative investigations of SON distribution along the surface of poled glass structures, but not suitable for quantitative measurements of SON tensor components.

\section{NORMAL REFLECTION MODE}

Direct observation of the depth distribution of the SON is obtained by $\mathrm{SH}$ imaging of cleaved edges of the samples. ${ }^{9}$ In this case SHSOM is used at normal incidence with a $40 \times$ microscope objective for focusing and collimating the light. Figure 5 shows simultaneous recordings of the reflected FH and SH radiation when the focused FH beam is scanned across the glass layer at different places on sample S3. The incident light was circularly polarized (in order to avoid backreflection into the laser) and the detected light was polarized in the direction perpendicular to the glass/air and glass/Si interfaces [along the $z$ axis in Fig. 1(b)].

Figure 5(a) shows a scan using a pump wavelength of $860 \mathrm{~nm}$. A convolution fit of a Gaussian beam profile and 
step function changes in linear reflectivity gives the beam diameter and the positions of the glass/air and glass/Si interfaces. ${ }^{9}$ From this fit the total width of the glass layer is found to be $8.8 \pm 0.2 \mu \mathrm{m}$, a value which is in reasonable agreement with the layer thickness determined previously. ${ }^{9}$ The fit gives a beam diameter of $2.0 \pm 0.1 \mu \mathrm{m}$ and a ratio of 11.4 for the FH reflectivities at the two interfaces. The latter corresponds quite well to the value of 9.7 obtained from the refractive index data. ${ }^{14}$

With the beam diameter determined, it is now possible to analyze the SH signal scan. The main feature is a peak in the center part of the glass layer, but nonvanishing signals are also observed both in Si and in the glass. The SH signal can thus be related to background nonlinear polarizabilities in the glass and in $\mathrm{Si}$ in addition to a $1.6 \pm 0.1 \mu \mathrm{m}$ wide region with the presumably constant SON representing the poled layer. The obtained width of the SON layer is less than the width of the Ge-doped glass layer. Furthermore, the peak of the $\mathrm{SH}$ signal is placed close to the boundary of the Ge-doped layer closest to the air/glass interface of the sample. ${ }^{9}$ It should be mentioned that, with the present spatial resolution of the SHSOM, it is impossible to determine which of the two SON profiles, i.e., the rectangular profile used here or the Gaussian one used previously, ${ }^{9}$ ensures a better fit to the experimental data.

For a FH wavelength of $760 \mathrm{~nm}$, large SH signals are also found to be located at the glass/air and glass/Si interfaces as seen in Figs. 5(b) and 5(c) recorded, respectively, inside and outside the poled region. The corresponding SH frequency is close to the direct band gap of $\mathrm{Si}$ and a resonance in SHG. ${ }^{15}$ This results in a more efficient surface SHG from $\mathrm{Si}$ at 760 than at $860 \mathrm{~nm}$ pump wavelength. The presence of the glass/Si interface reduces the symmetry and thus enhances the SH radiation polarized in the direction perpendicular to that interface. ${ }^{2}$ On the other hand, it is somewhat surprising that both the (unpoled) glass and Si surfaces produce nonzero SH signals at normal incidence [Fig. 5(c)]. The Si wafer is cleaved along a (100) plane and should not give rise to SHG at normal incidence. ${ }^{12}$ Neither should the surface of an amorphous material such as glass. We believe that SHG at normal incidence is due to the strong focusing of the pump beam, which creates a complicated field distribution with all field components being nonzero. ${ }^{16}$ The field component normal to the surface would then lead to SHG from isotropic media because of the field gradient in the surface layer ${ }^{17}$ Furthermore, in the case of $\mathrm{Si}$, it has been shown that a few degrees miscut of the surface leads to enhancement of SHG due to the appearance of atomic steps on the surface. ${ }^{18}$ These steps reduce the symmetry, thus allowing the surface to have in-plane SH polarization. Such effects are enhanced near the resonance in $\mathrm{SHG}$ from $\mathrm{Si},{ }^{15}$ as seen by comparison of Figs. 5(a) and 5(b). With respect to the unpoled glass surface, no clear dispersion of SHG was observed in the investigated wavelength range, and the absence or presence of the peak in the SH signal at the glass/air interface is determined by the absence or presence of surface defects (cf. Fig. 6 and the accompanying discussion).

The FH signal scans in Figs. 5(b) and 5(c) have been fitted with beam diameters of $1.8 \pm 0.1$ and $1.5 \pm 0.1 \mu \mathrm{m}$, re- spectively. These values are smaller than the ones obtained for wavelength of $860 \mathrm{~nm}$, as expected. Note that, for a Gaussian beam at $860 \mathrm{~nm}$ focused with an objective having a numerical aperture of 0.65 , the diameter of the beam waist can be as small as $\sim 0.84 \mu \mathrm{m}$. The above difference in beam diameters is caused by variations in focus adjustment. We found that the most convenient way to adjust the distance between the focusing objective and the sample surface is by looking for a maximum $\mathrm{SH}$ signal from $\mathrm{Si}$, i.e., from a region where the signal is independent of the beam position in the scanning plane.

In order to describe the $\mathrm{SH}$ signals at the glass boundaries, delta-function SON profiles are introduced at the two interfaces of the glass layer. In the fit shown in Fig. 5(b), the poled layer is represented by a $2.7 \pm 0.1 \mu \mathrm{m}$ wide rectangular SON profile. Like in the scan shown in Fig. 5(a), the SON layer is centered close to the boundary of the doped glass layer from the side of the air/glass interface. The widths obtained from the two scans are, however, somewhat different. This may reflect real variations in the thickness of the SON layer, for instance, due to variations in the poling field (see Sec. III) and/or inhomogeneities in the composition of the glass. Furthermore, since the actual beam profile varies near the beam waist, the SON layer width determined from the fits described above depends on the focusing of the pump beam. It should, however, be stressed that the comparison of the widths of SH contributions from the interfaces of the glass layer to that of the poled layer provides direct evidence of the fact that the SON layer created by poling has a finite thickness.

It should be noted that, even though in the depth scans [Fig. 5(b)] the SH signals at the interfaces of the glass layer are comparable to that originating from the poled layer, the nonlinearities at the interfaces do not contribute substantially to the SH signal in the transmission reflection mode. In this mode, the SH signal accumulated through the poled layer is several orders of magnitude larger than the signal generated in reflection from a few atomic layers at the glass/Si interface.

Figure 6 shows the $\mathrm{FH}$ and $\mathrm{SH}$ images of a 22 $\times 22 \mu \mathrm{m}^{2}$ area covering the glass layer and its interfaces to air and Si. The SH image reveals the SON distribution while the FH image clearly identifies the positions of the two interfaces. A fairly homogeneous distribution of the detected $\mathrm{SH}$ signal is seen along the poled layer and the glass/Si interface, disturbed only at a few points, probably due to surface defects. The variation along the glass/air interface, on the other hand, shows a number of peaks with a vanishing SH signal in between. We conclude that the SH signal at the glass/air interface is likely to be caused by defects on the free surface of the sample. These defects may be created during cleaving of the sample or they may be impurities left on the glass surface during the fabrication process. Surface defects might in turn introduce localized stresses in glass and scatter the pump FH field, creating strong gradients of all field components. Consequently, enhancement of SHG is expected when the focused FH beam illuminates such a defect.

Surface defects are generally found to give interesting distributions of the SH signal. Figure 7 shows FH and $\mathrm{SH}$ 

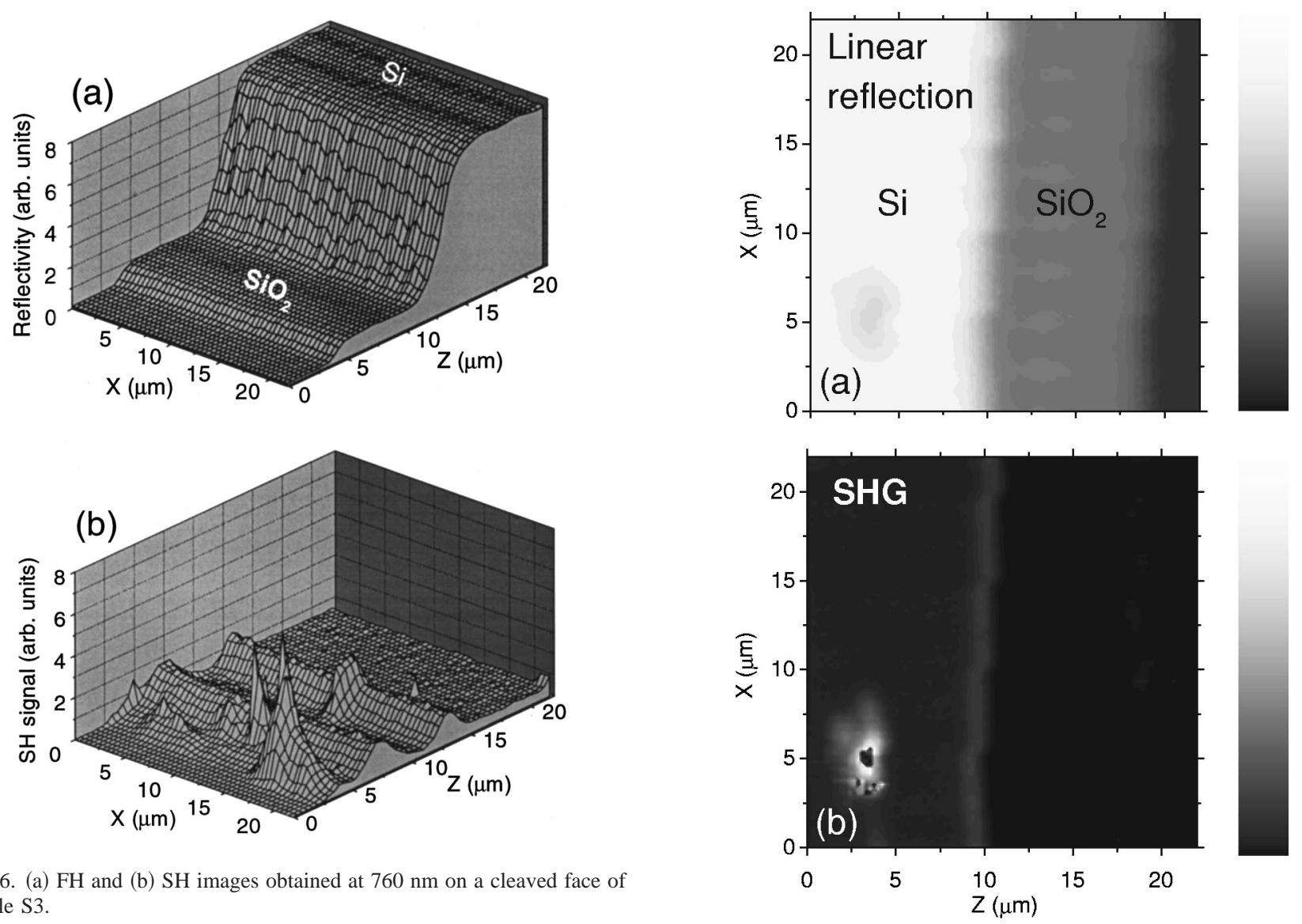

FIG. 6. (a) FH and (b) SH images obtained at $760 \mathrm{~nm}$ on a cleaved face of sample S3.

images recorded outside the poled area. In addition to the expected signal at the glass/Si interface, a region with a clear structure in both linear and nonlinear signals is seen on the $\mathrm{Si}$ surface. The FH signal has a valley with largely circular symmetry where the signal is about $30 \%$ lower than the signal from the surrounding $\mathrm{Si}$ surface. The corresponding $\mathrm{SH}$ signal shows a ring-shaped structure with high contrast to the low signal level inside the structure and in the surroundings. The variation in the SH signal can be qualitatively understood from the local macroscopic symmetry of the linear reflection. At the center of the valley in the FH image, the system has (approximately) full rotational symmetry, at least, the reflectivity increases in all directions. Thus the SON induced by this defect vanishes in the direction parallel to the surface. Conversely, on the slopes of the valley the rotational symmetry is broken, and in-plane nonlinear polarization is expected. The images shown in Fig. 7 also demonstrate the enhanced spatial resolution of SHSOM compared to linear microscopy. Notice that the diameter of the ring-shaped structure in the SH image is about $2 \mu \mathrm{m}$, and micron-size features in the SH image are clearly seen.

\section{CONCLUSIONS}

The results presented in this article have demonstrated the unique capabilities of SHSOM with respect to local probing of SON in poled glasses. Scanning of the sample from the top in oblique transmission reflection mode has been found to be well suited for testing the outcome of processes leading to integrated optical components. We have, however,
FIG. 7. (a) FH and (b) SH images illustrating the influence of the macroscopic symmetry of defects on the spatial distribution of $\mathrm{SH}$ radiation. The images were recorded outside the poled region of sample S3.

concluded that it is difficult to extract detailed quantitative information from the SH images obtained in this mode, since the SH signal recorded depends on geometrical parameters of the structure that may not all be well known. We have also demonstrated that detailed information about the SON distribution in depth can be obtained from scans on surfaces cleaved through the area of interest. Parallel recording of the FH and SH signals has been shown to facilitate the location of the SON layer relative to the glass interfaces within an accuracy of $\sim 0.2 \mu \mathrm{m}$. Overall, we believe that in combination with other techniques such as electron microscopy and secondary ion mass spectroscopy, SHSOM is very well suited for investigations of details of the poling process such as the dependence on chemical composition, temperature, poling field, etc.

We have also demonstrated that SHG is very sensitive to structures that break the in-plane symmetry of surfaces. The examples given in the present work are the glass/Si interface and surface defects. It has been shown that the SH images of the spatial distribution of the nonlinear signal reveal the macroscopic symmetry of surface defects. We believe that the SHSOM can be advantageously used for studies of surface inhomogeneities, e.g., semiconductor quantum dots and wires. 


\section{ACKNOWLEDGMENT}

Two of the authors (K.P. and S.I.B.) gratefully acknowledge financial support from the Danish Natural Science Research Council under Contract No. 9901971.

${ }^{1}$ R. W. Boyd, Nonlinear Optics (Academic, London, 1992).

${ }^{2}$ T. F. Heinz, in Nonlinear Surface Electromagnetic Phenomena, edited by H. Ponath and G. Stegeman (Elsevier, Amsterdam, 1991), p. 353.

${ }^{3}$ G. T. Boyd, Y. R. Shen, and T. W. Hänsch, Opt. Lett. 11, 97 (1986).

${ }^{4}$ S. Kurimura and Y. Uesu, J. Appl. Phys. 81, 369 (1997); Y. Uesu, S. Kurimura, and Y. Yamamoto, Appl. Phys. Lett. 66, 2165 (1995).

${ }^{5}$ S. I. Bozhevolnyi, J. M. Hvam, K. Pedersen, F. Laurell, H. Karlsson, T. Skettrup, and M. Belmonte, Appl. Phys. Lett. 73, 1814 (1998).

${ }^{6}$ V. Kirilyuk, A. Kirilyuk, and Th. Rasing, Appl. Phys. Lett. 70, 2306 (1997).

${ }^{7}$ M. Flörsheimer, Phys. Status Solidi A 173, 15 (1999).
${ }^{8}$ J. Vidra and M. Eich, Appl. Phys. Lett. 72, 275 (1998).

${ }^{9}$ J. Arentoft, K. Pedersen, S. I. Bozhevolnyi, M. Kristensen, P. Yu, and C. B. Nielsen, Appl. Phys. Lett. 76, 25 (2000).

${ }^{10}$ C. V. Poulsen, T. Storgaard-Larsen, J. Hübner, and O. Leistiko, Proc. SPIE 2998, 132 (1997).

${ }^{11}$ M. Cernusca, M. Hofer, and G. A. Reider, J. Opt. Soc. Am. B 15, 2476 (1998).

${ }^{12}$ J. E. Sipe, J. Opt. Soc. Am. B 4, 481 (1987).

${ }^{13}$ Y. R. Shen, The Principles of Nonlinear Optics (Wiley, New York, 1984).

${ }^{14}$ D. N. Nikogosyan, Properties of Optical and Laser-Related Materials: A Handbook (Wiley, Chichester, 1997).

${ }^{15}$ W. Daum, H.-J. Krause, U. Reichel, and H. Ibach, Phys. Rev. Lett. 71, 1234 (1993)

${ }^{16}$ M. Mansuripur, J. Opt. Soc. Am. A 3, 2086 (1986).

${ }^{17}$ R. W. Terhune and D. A. Weinberger, J. Opt. Soc. Am. B 4, 661 (1987).

${ }^{18}$ C. W. van Hasselt, M. A. Verheijen, and Th. Rasing, Phys. Rev. B 42, $9263(1990)$ 